\title{
Estenosis de la arteria renal unilateral de diagnóstico neonatal
} Neonatal diagnosis of unilateral renal artery stenosis

\author{
Dra. Sara M. Barbed Ferrández ${ }^{a}$, Dra. Inés Martínez Redondo ${ }^{a}$, Dra. Itziar Serrano Viñuales ${ }^{a}$, \\ Dra. Cristina Fernández Espuelas ${ }^{b}$, Dra. Yolanda Romero Salas ${ }^{c}$ y Dra. Cristina Gutiérrez Alonso ${ }^{d}$
}

\begin{abstract}
RESUMEN
La estenosis de la arteria renal es una causa rara de hipertensión arterial neonatal de origen renovascular. Hay muy pocos casos descritos en la literatura en esta etapa. La mayor parte de los pacientes con esta afectación permanecen asintomáticos, y la hipertensión se detecta en las revisiones pediátricas rutinarias. El diagnóstico puede realizarse mediante la combinación de hallazgos bioquímicos y radiológicos. El manejo inicial del paciente se basa en terapia farmacológica hasta alcanzar un crecimiento adecuado para evaluar la reparación definitiva de la lesión vascular o la nefrectomía en caso de supresión funcional del riñón afecto.

Se presenta a una paciente femenina recién nacida a término, con hipertensión arterial e importante falla cardíaca congestiva, originada por una estenosis unilateral de la arteria renal, con supresión funcional y atrofia del riñón afecto, que precisó importante soporte inotrópico y antihipertensivo durante los primeros días de vida, con importante mejoría clínica posterior. Palabras clave: estenosis de la arteria renal, hipertensión, hipertensión renovascular, recién nacido.
\end{abstract}

\begin{abstract}
Renal artery stenosis represents a rare cause of neonatal arterial hypertension of renovascular origin, having been described few cases in the literature at this stage of life. Most patients with this disease remain asymptomatic; hypertension can be detected in routine pediatric revisions. Diagnosis can be performed by combining biochemical and radiological findings. The initial management consists of pharmacological therapy in order to achieve adequate growth. Subsequently, it is necessary to assess definitive repair of the vascular lesion or nephrectomy in the case of functional abolition of the affected kidney.

We present a term newborn female, with arterial hypertension and an important congestive heart failure, caused by a unilateral renal artery stenosis, with functional abolition and atrophy of the affected kidney, which required an important inotropic and antihypertensive support during her first days of life, with significant clinical improvement subsequently.
\end{abstract}
a. Residencia de Pediatría.
b. Servicio de Neonatología.
c. Servicio de Nefrología Pediátrica.
d. Servicio de Radiología Pediátrica.
Hospital Universitario Materno-infantil Miguel Servet,
Zaragoza, España.

Correspondencia:

Dra. Sara M. Barbed Ferrández: sarabarbed@gmail.com

Financiamiento: Ninguno.

Conflicto de intereses: Ninguno que declarar.

Recibido: $18-12-2017$

Aceptado: 24-5-2018
Key words: renal artery stenosis, hypertension, renovascular hypertension, infant newborn.

http: / / dx.doi.org/10.5546/aap.2018.e675

Cómo citar: Barbed Ferrández SM, Martínez Redondo I, Serrano Viñuales I, et al. Estenosis de la arteria renal unilateral de diagnóstico neonatal. Arch Argent Pediatr 2018;116(5):e675-e678.

\section{INTRODUCCIÓN}

La hipertensión arterial presenta una incidencia del 1-2,5\% en recién nacidos ingresados en Unidades de Cuidados Intensivos, ${ }^{1}$ por lo que se trata de una patología diagnosticada de forma infrecuente en esta etapa de la vida. Se han descrito numerosas causas en este período; en la mayor parte de los casos, son de origen posnatal. Las más frecuentemente descritas son enfermedad renovascular, displasia broncopulmonar, enfermedad renal parenquimatosa y la iatrogenia.

La estenosis de la arteria renal es una causa rara de hipertensión neonatal de origen renovascular. Se han descrito muy pocos casos en la literatura en esta etapa de la vida. ${ }^{2,3}$ Esto se debe a que la mayor parte de los pacientes con estenosis de la arteria renal permanecen asintomáticos, y la hipertensión se detecta como hallazgo casual en las revisiones pediátricas rutinarias. ${ }^{4}$

El diagnóstico de esta entidad puede llevarse a cabo mediante la combinación de hallazgos bioquímicos y radiológicos. La estenosis de la arteria renal es capaz de estimular de forma muy activa el sistema renina-angiotensina-aldosterona y se puede detectar en una muestra de sangre periférica. Sin embargo, esto se ha estimado que ocurre en un $50-80 \%$ de los pacientes. ${ }^{5}$ Además, la ultrasonografía renal con doppler, que constituye la modalidad de imagen de elección en el estudio de la hipertensión neonatal, presenta, para el diagnóstico de esta entidad, una sensibilidad del 50-98\% y una especificidad del $60-98 \%$. Permite visualizar directamente la arteria renal y examinar su permeabilidad mediante el modo doppler-color. ${ }^{6}$ Una vez detectada, hay que tener en cuenta que esta entidad puede asociarse con 
coartación aórtica media y estenosis vasculares cerebrales, que es preciso descartar. ${ }^{4}$ También es necesario tener en cuenta que esta alteración se ha descrito asociada a diversos síndromes (por ejemplo, neurofibromatosis, Williams, KlippelTrenaunay-Weber), a ciertas infecciones, como la rubeola congénita, a compresión extrínseca o, de forma más frecuente, a displasia fibromuscular ${ }^{1}$ y ha estado implicada, en un 65-95\% de los casos, como causa de la lesión estenótica. ${ }^{4}$

En cuanto al tratamiento, inicialmente, suele ser necesario manejar al paciente con terapia farmacológica hasta alcanzar un crecimiento adecuado para evaluar la reparación definitiva de la lesión vascular o realizar la nefrectomía en caso de supresión funcional del riñón afecto. ${ }^{7}$

Se presenta el caso de una paciente femenina recién nacida a término, con hipertensión arterial e importante falla cardíaca congestiva, originada por una estenosis unilateral de la arteria renal.

\section{EXPOSICIÓN DEL CASO CLÍNICO}

Paciente femenina recién nacida, tras un parto vaginal instrumentado con ventosa por la no progresión, a las 37 semanas de edad gestacional, con peso de nacimiento de 3830 gramos (peso superior al percentil 95, elevado para su edad gestacional) y un puntaje en el test de Apgar 9 al minuto y 10 a los 5 minutos del nacimiento. Era la primera hija de padres no consanguíneos, cuya madre presentaba diabetes mellitus tipo 1. El embarazo fue controlado y se precisó el ingreso materno por un episodio de cetoacidosis diabética en la semana 30 de gestación, sin observarse riesgo de pérdida de bienestar fetal ni otras alteraciones.

Al nacer, ingresó en Cuidados Neonatales por hipoglucemias mantenidas, que precisaron el aporte de glucosa por vía intravenosa. Mientras permanecía hospitalizada, se detectaron cifras elevadas de presión arterial, de forma intermitente. Sin embargo, a los 9 días de vida, tuvo que ser referida a una Unidad de Cuidados Intensivos de un centro de referencia, debido a un empeoramiento clínico brusco, con elevación persistente de las cifras tensionales por encima del p95 para su edad gestacional (tensión arterial sistólica mayor de $90 \mathrm{mmHg}$ y diastólica mayor de $60 \mathrm{mmHg}$ ) y signos clínicos de repercusión hemodinámica (taquicardia, mala perfusión periférica, reducción del ritmo de diuresis).

A su llegada, fue monitorizada y estabilizada, y se inició, posteriormente, la evaluación del grado de repercusión hemodinámica y el estudio de su cuadro hipertensivo. Se realizó la analítica sanguínea y de orina completa, el estudio ecocardiográfico y la ecografía abdominal. En la ecocardiografía, presentó una fracción de eyección del ventrículo izquierdo del 30\%, insuficiencia mitral y tricúspide moderadas e hipertensión pulmonar moderada-grave. En la ecografía abdominal, se observó estenosis de la arteria renal derecha, de aspecto crónico, acompañada de atrofia renal derecha y crecimiento renal izquierdo compensador (Figura 1). Los resultados obtenidos en el análisis de sangre apoyaron este diagnóstico, con un sistema renina-angiotensinaaldosterona con índices elevados (aldosterona: $1356 \mathrm{pg} / \mathrm{ml}$; valores de referencia: $15-133 \mathrm{pg} /$ $\mathrm{ml}$; actividad de renina plasmática: $271,6 \mathrm{ng} / \mathrm{ml} /$ hora; valores de referencia: $0,32-1,84 \mathrm{ng} / \mathrm{ml} /$ hora) y cifras de prohormona N-terminal del péptido natriurético cerebral (NT-ProBNP; biomarcador de insuficiencia cardíaca y antagonista natural del sistema renina-angiotensina-aldosterona) elevadas, con una cifra máxima de $98000 \mathrm{pg} / \mathrm{ml}$ (valores de referencia: 0-300 pg/ml).

El estado clínico de la paciente requirió iniciar el soporte inotrópico con milrinona (inhibidor de la fosfodiesterasa III) y el tratamiento antihipertensivo con nitroprusiato (vasodilatador directo arteriolar y venoso), captopril (inhibidor de la enzima convertidora de angiotensina) y bolos puntuales de hidralazina (vasodilatador directo). Presentó un progresivo control de las cifras tensionales, con mejoría hemodinámica secundaria. A las 48 horas, se asoció amlodipina (antagonista selectivo de los canales de calcio) y se incrementó la dosis de captopril, por lo que se pudo retirar el soporte con milrinona y nitroprusiato.

Se realizaron controles ecocardiográficos seriados, con mejoría progresiva de la función ventricular hasta normalizarse, y presentó hipertrofia del ventrículo izquierdo residual.

Cuando la paciente se encontraba estable, se prosiguió el estudio etiológico y se realizó una gammagrafía renal, que mostró la supresión funcional del riñón derecho, con el riñón izquierdo moderadamente aumentado de tamaño, con captación de radiotrazador normal (Figura 2). Se realizó, asimismo, un estudio oftalmológico con el objetivo de mostrar la repercusión sistémica del cuadro, que mostró una retinopatía hipertensiva grado III.

Debido a su mejoría clínica, la paciente fue dada de alta a los 28 días de vida con un tratamiento antihipertensivo por vía oral y 
controles ambulatorios en consultas de Nefrología Infantil y Neonatología.

A los 15 meses de vida, la paciente se encontraba sin tratamiento antihipertensivo desde los 12 meses y mantenía cifras de tensión arterial dentro de los percentiles adecuados para su edad.

Figura 1. Asimetría renal manifiesta, con riñón derecho disminuido de tamaño (A. Riñón izquierdo; B. Riñón derecho). Arterias renales (C. Arteria renal izquierda; D. Arteria renal derecha) permeables en el origen, de calibre normal; la derecha presenta disminución de su calibre en el tercio medio (véase el calibre señalado con flechas blancas). Los hallazgos sugieren estenosis de la arteria renal derecha, de aspecto crónico, con atrofia renal derecha y crecimiento renal izquierdo compensador.

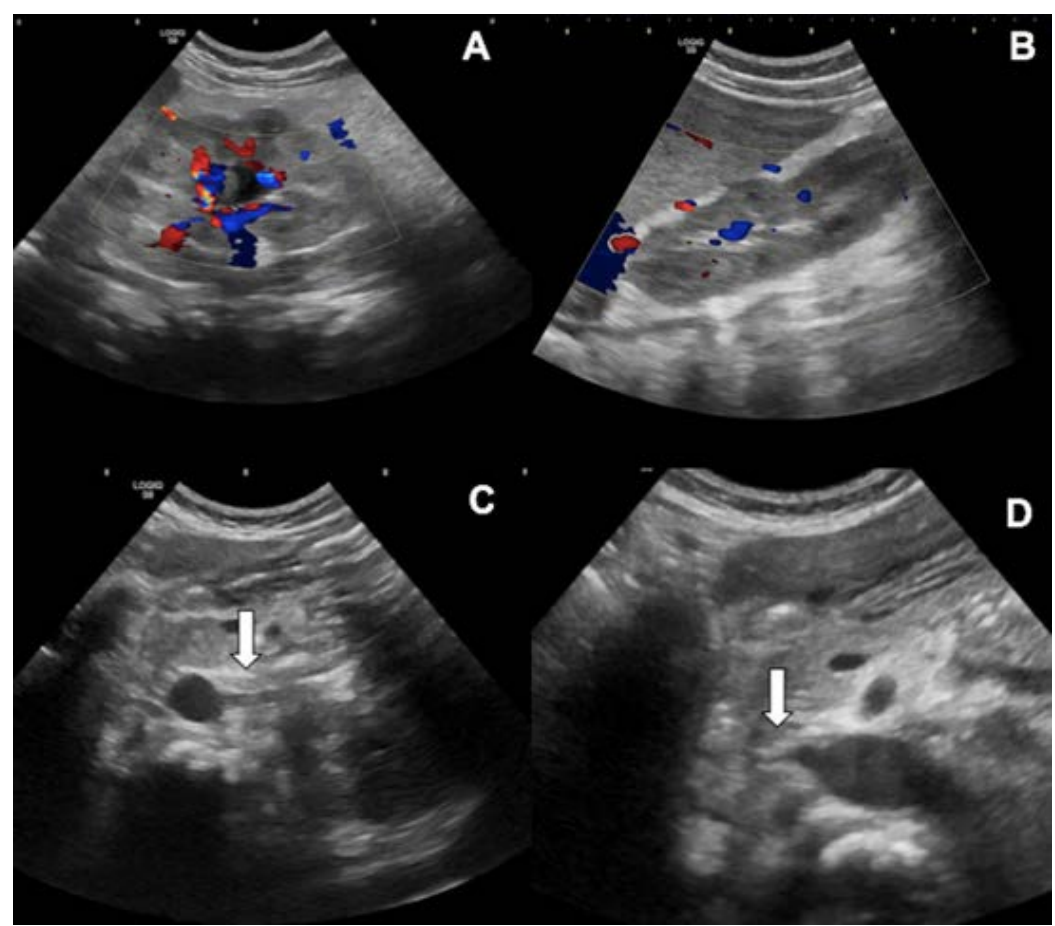

FIGURA 2. Supresión funcional del riñón derecho. Riñón izquierdo moderadamente aumentado de tamaño con captación normal de radiotrazador (flecha negra)

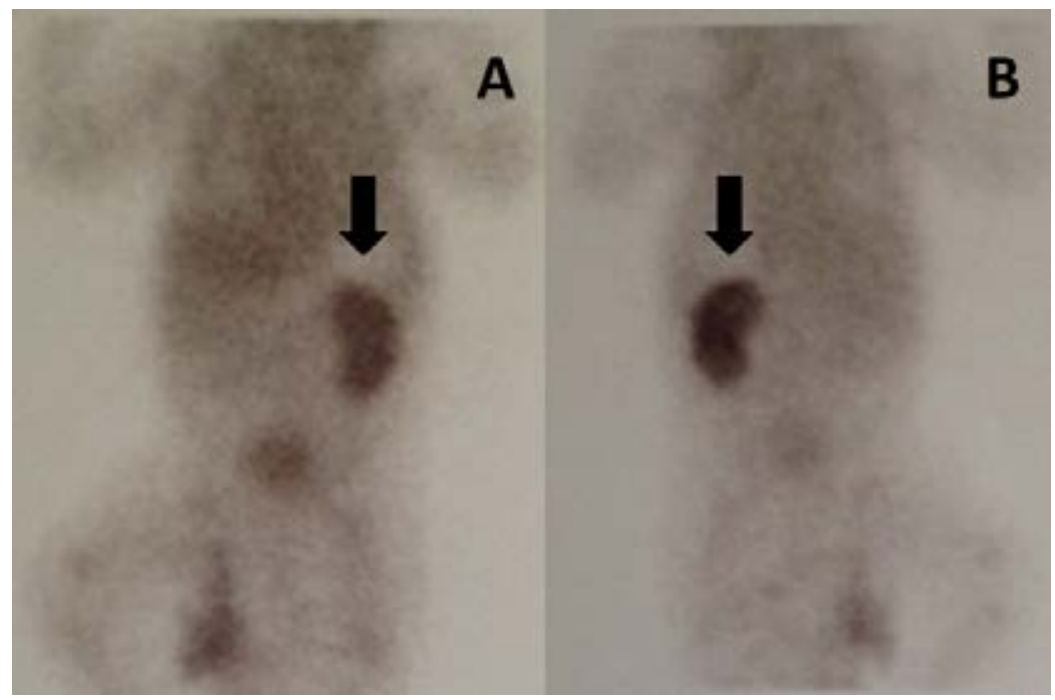

A. Imagen anteroposterior. B. Imagen posteroanterior. 
Los controles ecocardiográficos revelaron una leve hipertrofia ventricular izquierda, sin repercusión en la actualidad, y la retinopatía hipertensiva se resolvió sin tratamiento. La ecografía abdominal seguía mostrando un riñón derecho atrófico, con señal doppler-color disminuida y registros de alta resistencia, por lo que continuaba siendo controlada de forma ambulatoria, pendiente de una posible nefrectomía unilateral derecha definitiva.

\section{COMENTARIO FINAL}

El caso de nuestra paciente presenta ciertas particularidades. Por una parte, el hecho de que se observaran cifras tensionales elevadas desde el primer momento en el que se monitorizó, junto con la importante repercusión hemodinámica y el hallazgo de hipertrofia ventricular izquierda, indica que el daño podría tener un origen crónico, posiblemente, como resultado de hipertensión arterial en la etapa fetal, como ocurre en algunos casos descritos previamente. ${ }^{2,3}$ A pesar de no haber encontrado una clara relación en la literatura, dentro de los pocos casos publicados de hipertensión arterial neonatal de causa renovascular, en algunos, existe el antecedente de diabetes materna, por lo que sería interesante continuar la investigación en estudios futuros. ${ }^{2}$

La presión arterial no se mide de forma rutinaria en los niños antes de la edad de 3 años. En consecuencia, la hipertensión arterial grave, raramente, se reconoce antes de esta edad, a menos que ocasione síntomas de forma temprana, ${ }^{8}$ como ocurrió con nuestra paciente, lo que facilitó la realización de un diagnóstico de forma precoz.

Por otra parte, la estenosis de la arteria renal, en esta etapa de la vida, presenta un particular reto debido al cuadro clínico de aparición brusca con empeoramiento progresivo y al difícil manejo antihipertensivo.

Un estudio publicado, ${ }^{8}$ en el que se realizó el seguimiento en una serie de 5 pacientes con diagnóstico de estenosis de la arteria renal durante su primer año de vida, muestra que todos ellos presentaron síntomas de falla cardíaca congestiva con hipertrofia ventricular izquierda y fueron tratados con terapia farmacológica hasta los 4,4 $\pm 0,9$ años, con corrección quirúrgica posterior en todos los casos. Se mantuvieron niveles tensionales por encima del percentil 95 para su edad durante el tratamiento farmacológico en 4 de los 5 casos, con posterior normalización en todos ellos tras la reparación vascular, durante un seguimiento de 9,4 \pm 2 años.

Sin embargo, nuestra paciente se encontraba asintomática a los 15 meses de vida. Seguía con controles seriados en consultas de Nefrología para continuar el seguimiento tanto de la tensión arterial como de la función renal, con cifras tensionales mantenidas en percentiles adecuados, sin precisar el tratamiento farmacológico antihipertensivo y sin haber recibido tratamiento quirúrgico definitivo mediante nefrectomía de su riñón derecho con supresión funcional.

Esto contrasta con los casos previos publicados, en los que se precisó la administración del tratamiento farmacológico hasta la posibilidad de realizar la reparación vascular o la nefrectomía definitiva. ${ }^{2,3,8}$

\section{REFERENCIAS}

1. Flynn JT. Etiology, clinical features, and diagnosis of neonatal hypertension. UpToDate. [Acceso: 13 denoviembre de 2017]. Disponible en: https://www.uptodate.com/ contents / etiology-clinical-features-and-diagnosis-ofneonatal-hypertension.

2. Hegde S, Wright C, Shenoy M, et al. Renovascular hypertension commencing during fetal life. Arch Dis Child Fetal Neonatal Ed. 2007; 92(4):F301-4.

3. Wilson DI, Appleton RE, Coulthard MG, et al. Fetal and infantile hypertension caused by unilateral renal arterial disease. Arch Dis Child. 1990; 65(8):881-4.

4. Robinson L, Gedroyc W, Reidy J, et al. Renal artery stenosis in children. Clin Radiol .1991; 44(6):376-82.

5. Wilcox CS. Use of angiotensin-converting-enzyme inhibitors for diagnosing renovascular hypertension. Kidney Int. 1993; 44(6):1379-90.

6. Fernández Hernández CM, Sarabia Tirado F, Ocete Ocete, A et al. Estenosis de arteria renal, diagnóstico y tratamiento por imagen. Radiología. 2014; 56(Espec Cong):1280.

7. Flynn JT. Neonatal hypertension: diagnosis and management. Pediatr Nephol 2000; 14(4):332-41.

8. Bendel-Stenzel M, Najarian JS, Sinaiko AR. Renal artery stenosis in infants: long-term medical treatment before surgery. Pediatr Nephol. 1996; 10(2):147-51. 